\title{
Combined Hepatocellular-Cholangiocarcinoma Associated With Radiofrequency Ablation for Hepatocellular Carcinoma
}

\author{
Susumu Takamatsu ${ }^{\mathrm{a}, \mathrm{b}}$, Hiroto Nagano ${ }^{\mathrm{a}}$, Shunro Ootsukasa ${ }^{\mathrm{a}}$, Yasuyuki Kawachi ${ }^{\mathrm{a}}$, \\ Hiroshi Maruyama ${ }^{\mathrm{a}}$
}

\begin{abstract}
The patient was a 70-year-old male with liver cirrhosis associated with viral hepatitis $\mathrm{C}$. He underwent radiofrequency ablation (RFA) for hepatocellular carcinoma (HCC) four times of in the 3 years prior to this reported event. Although he was closely followed after RFA by imaging every 3 - 4 months, a hypovascular and ill-defined tumor was revealed in segment IV of the liver by enhanced CT. The tumor expanded from the necrotic area associated with RFA performed 2 years prior. Because the tumor rapidly enlarged and was accompanied by a progressive tumor thrombus that filled the left portal vein, left hepatectomy was urgently performed. Histopathological examination of the resected specimen demonstrated that the tumor was mostly composed of moderately differentiated HCC. However, it also included obvious glandular structures with mucin production, which were immunohistochemically positive for both cytokeratin 7 and 19. These features are characteristic of cholangiocarcinoma; therefore the tumor was diagnosed as an uncommon combined hepatocellular-cholangiocarcinoma (cHCC-CC). From both pathological investigation and retrospective review of imaging results, the present $\mathrm{CHCC}-\mathrm{CC}$ was presumed to derive from a locally recurring tumor with morphologic changes to the biliary phenotype induced by RFA for HCC. Both distant and intrahepatic metastases appeared 4 months after surgery and the patient died of cancer 15 months after surgery in spite of anti-cancer treatments.
\end{abstract}

Keywords: Combined hepatocellular-cholangiocarcinoma; Radiofrequency ablation; Hepatocellular carcinoma; Local recurrence; Morphologic change; Biliary phenotype; Liver resection

\section{Introduction}

Combined hepatocellular-cholangiocarcinoma (cHCC-CC) is

\footnotetext{
Manuscript accepted for publication December 09, 2015

aDepartment of Surgery, Musashino Red Cross Hospital, 1-26-1, Kyounancho, Musashino-shi, Tokyo 180-8610, Japan

${ }^{b}$ Corresponding Author: Susumu Takamatsu, Department of Surgery, Musashino Red Cross Hospital, 1-26-1, Kyounan-cho, Musashino-shi, Tokyo 180-8610, Japan. Email: s.takamatsu@mac.com
}

doi: http://dx.doi.org/10.14740/jmc2387w defined as a tumor comprised of both hepatocellular carcinoma (HCC) and cholangiocarcinoma (CC) within the same tumor [1]. It is quite uncommon and accounts for only approximately $1 \%$ of primary liver cancers [2-5]. Although the pathogenesis of cHCC-CC remains uncertain, it has been recently suggested that cHCC-CC may derive from a hepatic stem cell or hepatic progenitor cell $[6,7]$.

Radiofrequency ablation (RFA) is established as an effective and less invasive treatment for small $\mathrm{HCC}$, and is thus accepted as an alternative to surgery [8]. However, local recurrence or local tumor progression after RFA remains a significant problem with a reported incidence ranging from $0.9 \%$ to $12 \%$ [8-14]. Among the cases with local recurrences, few demonstrate rapid and aggressive progression associated with morphologic changes [15-18].

We herein report a rare case of cHCC-CC, which is presumed to derive from a local recurrence with morphologic changes to the biliary phenotype caused by RFA for small HCC.

\section{Case Report}

The patient was a 70-year-old male with a 20 -year history of chronic liver disease associated with hepatitis $\mathrm{C}$ virus. He underwent RFA for HCCs three times between April 2007 and November 2009. Subsequently, an additional 1-cm tumor in segment IV of the liver showed arterial hyperenhancement and washout in the delayed phase of three-phase CT (Fig. 1). Because those enhancements agreed with the typical diagnostic characteristics of HCC, the tumor was diagnosed as a new HCC. Percutaneous RFA for this HCC was performed in July 2010 and complete necrosis was radiologically confirmed after RFA. He was subsequently monitored closely by imaging every 3 - 4 months. Slight intrahepatic bile duct dilatation, which was restricted adjacent to the necrotic area because of RFA, was observed by CT in November 2011 (Fig. 2). At that time, the finding was not interpreted as evidence of local recurrence. In May 2012, a hypovascular and ill-defined tumor, which expanded from necrotic area in segment IV of the liver, was revealed by CT (Fig. 3). Because the tumor rapidly enlarged and was accompanied by a progressive portal vein tumor thrombus that filled the left portal vein, left hepatectomy with removal of the portal vein tumor thrombus extending to the 

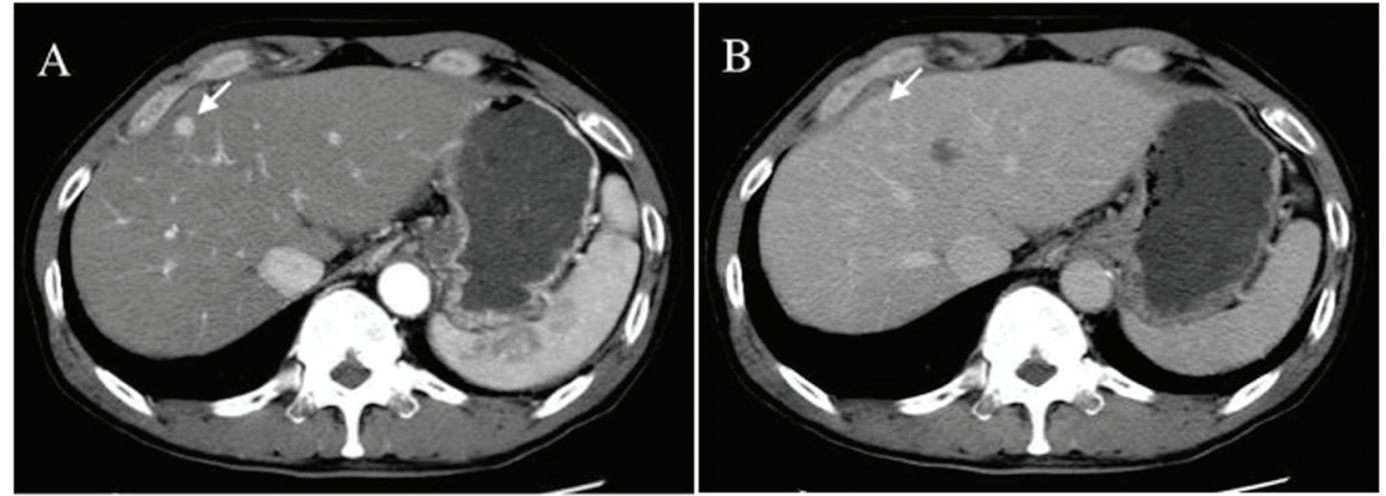

Figure 1. Three-phase CT in June 2010. A 1-cm tumor observed in segment IV of the liver. The tumor was diagnosed as hepatocellular carcinoma from classical enhancement on three-phase CT. The tumor (arrow) was revealed to be hypervascular on early phase (A), and washed out on delayed phase (B).
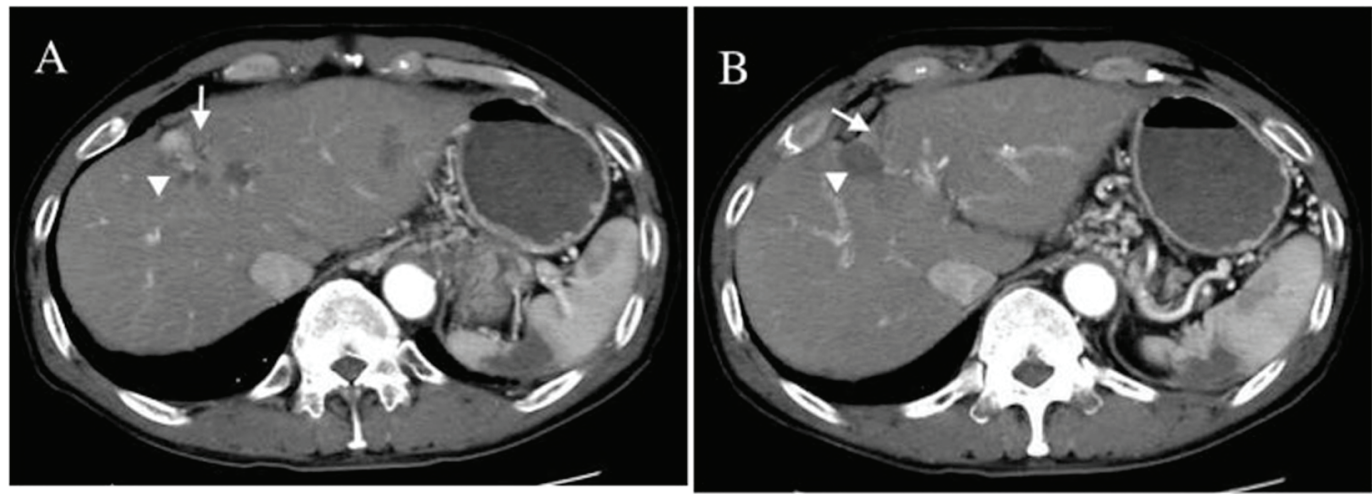

Figure 2. CT in November 2011. Slight bile duct dilatation (arrow) adjacent to necrotic area (arrowhead) caused by RFA.

portal trunk was performed urgently. In macroscopic findings of the resected specimen, a white and unencapsulated tumor showed infiltrative growth and was accompanied by tumor thrombus that filled the left portal vein (Fig. 4). Pathological examination showed that the tumor consisted of mostly moderately differentiated $\mathrm{HCC}$ and partly well to poorly differen- tiated HCC. Furthermore, obvious glandular structures with mucin production were observed (Fig. 5). Immunohistochemical investigation demonstrated that the glandular component was positive for both cytokeratin (CK) 7 and CK19 (Fig. 6) and was regarded as $\mathrm{CC}$; therefore, the tumor was diagnosed as cHCC-CC. The postoperative course was uneventful. Four
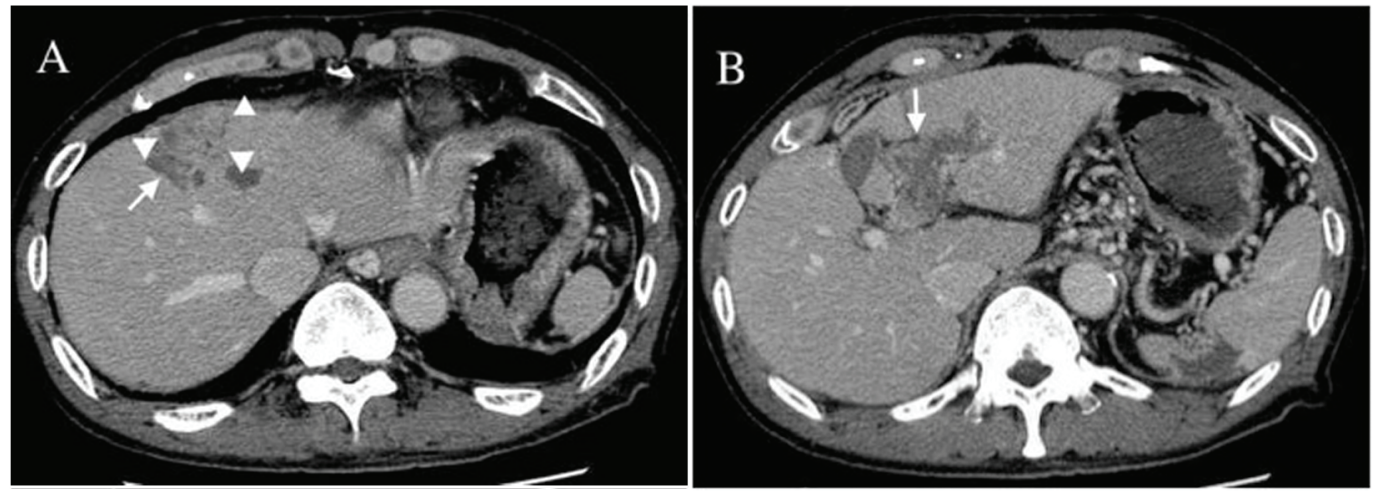

Figure 3. CT in May 2012. (A) An ill-defined tumor (arrowhead), which was adjacent to necrotic area (arrow) caused by RFA performed in July 2010, was demonstrated on delayed phase of CT. (B) CT depicted massive portal vein tumor thrombus (arrow) that filled left portal vein. 


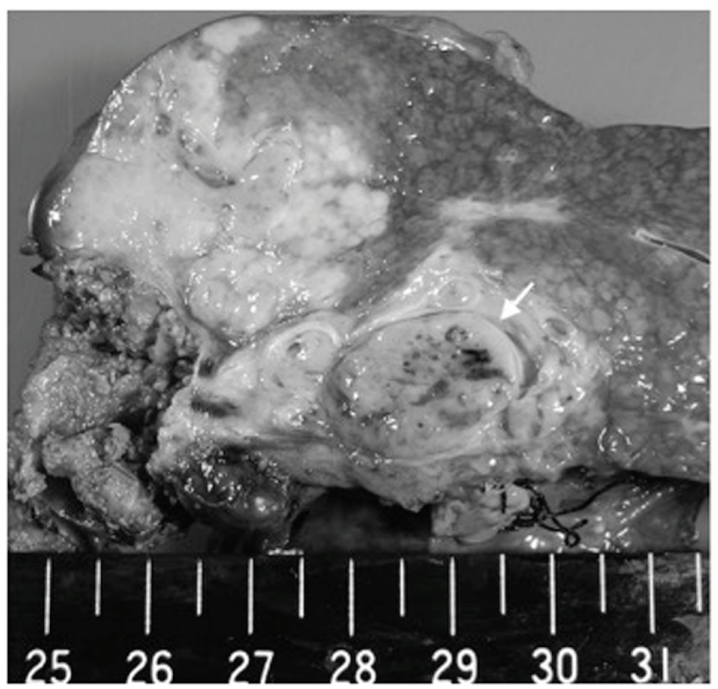

Figure 4. Macroscopic finding of the resected specimen. On a crosssection of resected specimen, there was a white and unencapsulated tumor with massive portal vein tumor thrombus (arrow) that filled the left portal vein. months after surgery, CT revealed multiple recurrent tumors in the remnant liver and distant metastases to lung and lymph nodes. Although the patient underwent systemic chemotherapy by orally administration of a standard dose of S-1 for recurrent disease, he died 15 months after surgery. Autopsy was not performed.

\section{Discussion}

RFA is established as an ablation therapy for HCC and is accepted as an alternative to surgical resection, especially for small HCC with impairment of hepatic functional reserve [8]. Nevertheless, local recurrence or local tumor progression remains a significant problem after RFA, and its incidence has recently been reported to range from $0.9 \%$ to $12 \%$ [8-14]. Local recurrence after RFA is generally considered to be due to regrowth of residual tumor cells. In fact, Pompili and colleagues [19] reported that only 47\% (14/30) of HCCs treated by RFA obtained complete necrosis in their retrospective study using explanted livers. Furthermore, they also showed that $50 \%$ of all ablated tumors were still viable on pathological ex-
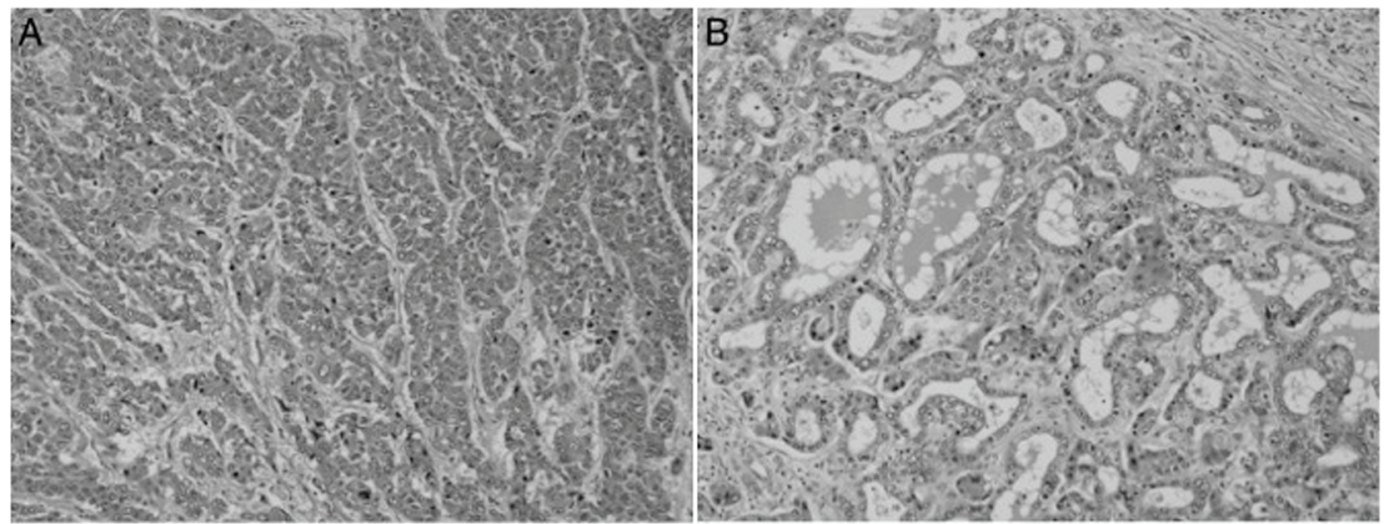

Figure 5. Microscopic findings of the resected tumor (hematoxylin \& eosin stain, $\times 100$ ). (A) The tumor was mainly composed of moderately differentiated hepatocellular carcinoma, revealing a trabecular pattern. (B) The tumor included obvious glandular structures with mucin production and was regarded as cholangiocarcinoma.
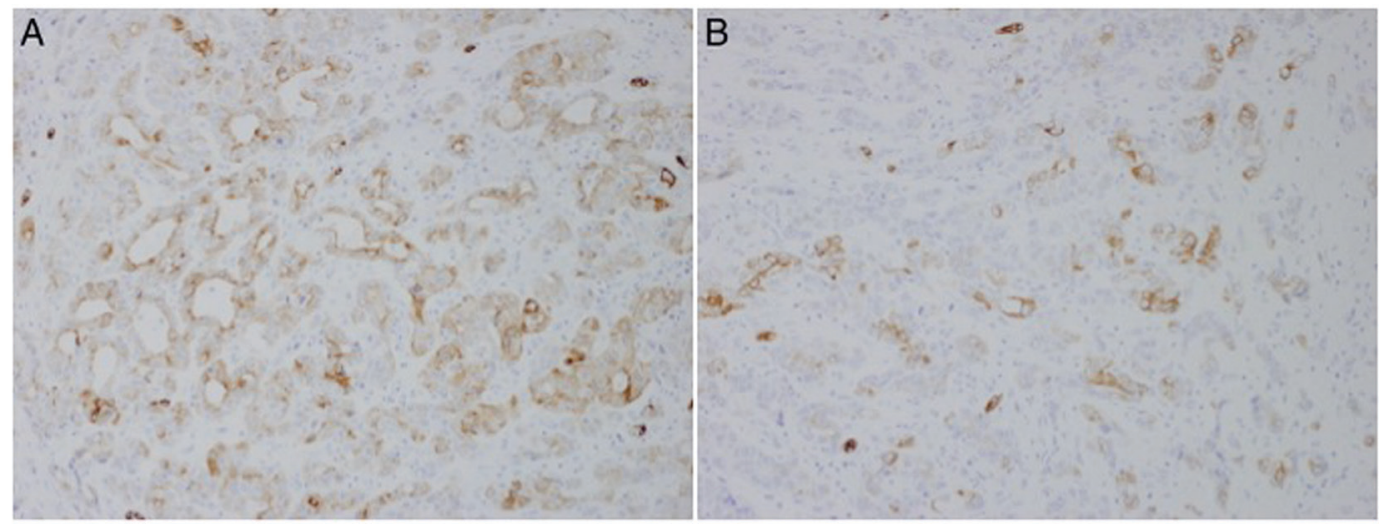

Figure 6. Immunohistochemical findings $(\times 100)$. The glandular structure in the present tumor was composed of the cells staining positive for both cytokeratin $19(\mathrm{~A})$ and cytokeratin $7(\mathrm{~B})$. 
amination, in spite of being assessed as achieving complete necrosis by CT before orthotopic liver transplantation.

The present case had already undergone RFA three times previously for HCCs that developed in cirrhotic liver associated with viral hepatitis C. A subsequent 1-cm tumor developed, which, by three-phase CT, showed two classic enhancements associated with HCC. It was clinically diagnosed as HCC, percutaneous RFA was performed, and it was assessed by imaging that complete necrosis had been achieved. Despite this, about 2 years later, a large tumor with a massive left portal vein tumor thrombus presented in the same segment of the liver. Although the resected specimen was macroscopically quite different from typical HCC, pathological investigation revealed that it mostly was composed of moderately differentiated HCC and surrounded necrotic tissue resulted from RFA. Therefore, the present tumor was presumed to be a locally recurring tumor after RFA for HCC.

Diagnosis of cHCC-CC is usually confirmed by the presence of both hepatocellular and cholangiocellular features by histopathological examination. Hepatocellular features are described as trabecular or pseudoglandular architecture of cells with prominent nucleoli, eosinophilic cytoplasm, and bile production, while cholangiocellular features include glandular structures with mucin production. Furthermore, immunohistochemical investigations have recently been established to verify the histopathological diagnosis. Namely, hepatocellular components express hepatocyte antigen (hepatocyte paraffin 1: Hep Par 1), CK8, and CK18; whereas cholangiocellular components are positive for CK7 and CK19 [5]. Although it has recently been suggested that $\mathrm{CHCC}-\mathrm{CC}$ originates from hepatic stem cells or hepatic progenitor cells $[6,7]$, the pathogenesis of cHCC-CC remains uncertain.

On retrospective review of imaging results over the course of the patient's follow-up, slight bile duct dilatation in the area adjacent to the RFA-induced necrosis was identified by CT at 16 months after treatment. Because bile duct dilatation peripheral to $\mathrm{CC}$ is often observed, this finding suggests that the $\mathrm{CC}$ component of this tumor already existed at that time. Hence we suspect that the pathogenesis of present $\mathrm{cHCC}-\mathrm{CC}$ was associated with a morphologic change from moderately differentiated HCC to CC. It has been previously reported that some local recurrences after RFA can exhibit phenotypic conversion or malignant transformation [15-18]. These reports implied that the thermal effect of RFA increased the malignant potential of the tumor [18]. The results of the experimental studies also confirmed that both malignant transformation and enhancement of malignant potential were induced by sublethal or insufficient RFA [20, 21]. Dong and co-workers [22] described that insufficient RFA might promote the epithelial-mesenchymal transition, which is considered to be a key process of metastasis of cancer. Although Nishihara et al [23] described that transcatheter arterial chemoembolization induced the biliary phenotype of HCC, there has been no previous report that RFA has an impact on acquiring the biliary phenotype of HCC. Therefore, the present $\mathrm{cHCC}-\mathrm{CC}$ is considered to be very rare.

The present $\mathrm{cHCC}-\mathrm{CC}$ recurred as systemic disease rapidly after surgery and consequently resulted in a poor prognosis. Among reported locally recurring tumors after locoregional therapy for $\mathrm{HCC}$, few demonstrated rapid and aggressive progression, and poor prognoses similar to the present case. Because it is important for recurring tumors that demonstrate malignant behavior to be identified early, close follow-up is necessary after locoregional therapy for HCC.

In conclusion, we herein report a rare case of $\mathrm{cHCC}-\mathrm{CC}$, which derived from the local recurrence of the tumor, accompanied by morphologic changes to the biliary phenotype caused by RFA for small HCC. Although RFA is an established and effective ablation therapy for small $\mathrm{HCC}$, local recurrence remains a significant problem. Among locally recurrent tumors, few demonstrate rapid and aggressive progression and poor prognoses. Morphologic changes induced by RFA are believed to be the cause of such a clinical course. Close followup is therefore important after locoregional therapy including RFA for HCC, even if complete necrosis is reported using imaging procedures.

\section{Disclosure}

This case was already reported in International Surgical Week 2013 in Helsinki, Finland.

\section{Grant Support}

There was no grant support or assistance.

\section{Conflict of Interest}

All of the authors declare that they have no conflicts of interest.

\section{References}

1. Wittekind C, Fischer HP, PonchonT. Combined hepatocellular carcinoma and cholangiocarcinoma. In: World Helth Organization Classification of tumors: Pathology and Genetics of Tumors of the Digestive System. Lyon, France: IARC Press. 2000;181.

2. Ikai I, Kudo M, Arii S, Omata M, Kojiro M, Sakamoto M, Takayasu K. The Liver Cancer Study Group of Japan. Report of the 18th follow-up survey of primary liver cancer in Japan. Hepatology Research. 2010;40:1043-1059.

3. Wachtel MS, Zhang Y, Xu T, Chiriva-Internati M, Frezza EE. Combined hepatocellular cholangiocarcinomas; analysis of a large database. Clin Med Pathol. 2008;1:4347.

4. Yin X, Zhang BH, Qiu SJ, Ren ZG, Zhou J, Chen XH, Zhou Y, et al. Combined hepatocellular carcinoma and cholangiocarcinoma: clinical features, treatment modalities, and prognosis. Ann Surg Oncol. 2012;19(9):28692876.

5. Singh S, Chakraborty S, Bonthu N, Radio S, Hussain SM, Sasson A. Combined hepatocellular cholangiocarcinoma: a case report and review of literature. Dig Dis Sci. 
2013;58(7):2114-2123.

6. Roskams T. Liver stem cells and their implication in hepatocellular and cholangiocarcinoma. Oncogene. 2006;25(27):3818-3822.

7. Zhang F, Chen XP, Zhang W, Dong HH, Xiang S, Zhang WG, Zhang BX. Combined hepatocellular cholangiocarcinoma originating from hepatic progenitor cells: immunohistochemical and double-fluorescence immunostaining evidence. Histopathology. 2008;52(2):224-232.

8. Livraghi T, Meloni F, Di Stasi M, Rolle E, Solbiati L, Tinelli C, Rossi S. Sustained complete response and complications rates after radiofrequency ablation of very early hepatocellular carcinoma in cirrhosis: Is resection still the treatment of choice? Hepatology. 2008;47(1):82-89.

9. Shiina S, Tateishi R, Arano T, Uchino K, Enooku K, Nakagawa H, Asaoka Y, et al. Radiofrequency ablation for hepatocellular carcinoma: 10-year outcome and prognostic factors. Am J Gastroenterol. 2012;107(4):569-577; quiz 578.

10. Choi D, Lim HK, Rhim H, Kim YS, Lee WJ, Paik SW, Koh KC, et al. Percutaneous radiofrequency ablation for early-stage hepatocellular carcinoma as a first-line treatment: long-term results and prognostic factors in a large single-institution series. Eur Radiol. 2007;17(3):684-692.

11. Lam VW, Ng KK, Chok KS, Cheung TT, Yuen J, Tung H, Tso WK, et al. Risk factors and prognostic factors of local recurrence after radiofrequency ablation of hepatocellular carcinoma. J Am Coll Surg. 2008;207(1):20-29.

12. N'Kontchou G, Mahamoudi A, Aout M, Ganne-Carrie N, Grando V, Coderc E, Vicaut E, et al. Radiofrequency ablation of hepatocellular carcinoma: long-term results and prognostic factors in 235 Western patients with cirrhosis. Hepatology. 2009;50(5):1475-1483.

13. Mulier S, Ni Y, Jamart J, Ruers T, Marchal G, Michel L. Local recurrence after hepatic radiofrequency coagulation: multivariate meta-analysis and review of contributing factors. Ann Surg. 2005;242(2):158-171.

14. Curley SA, Izzo F, Ellis LM, Nicolas Vauthey J, Vallone P. Radiofrequency ablation of hepatocellular cancer in 110 patients with cirrhosis. Ann Surg. 2000;232(3):381391.

15. Koda M, Maeda Y, Matsunaga Y, Mimura K, Murawaki Y, Horie Y. Hepatocellular carcinoma with sarcomatous change arising after radiofrequency ablation for welldifferentiated hepatocellular carcinoma. Hepatol Res. 2003;27(2):163-167.

16. Seki $T$, Tamai $T$, Ikeda $K$, Imamura $M$, Nishimura A, Yamashiki N, Nakagawa T, et al. Rapid progression of hepatocellular carcinoma after transcatheter arterial chemoembolization and percutaneous radiofrequency ablation in the primary tumour region. Eur J Gastroenterol Hepatol. 2001;13(3):291-294.

17. Portolani N, Tiberio GA, Ronconi M, Coniglio A, Ghidoni S, Gaverini G, Giulini SM. Aggressive recurrence after radiofrequency ablation of liver neoplasms. Hepatogastroenterology. 2003;50(54):2179-2184.

18. Takada Y, Kurata M, Ohkohchi N. Rapid and aggressive recurrence accompanied by portal tumor thrombus after radiofrequency ablation for hepatocellular carcinoma. Int J Clin Oncol. 2003;8(5):332-335.

19. Pompili M, Mirante VG, Rondinara G, Fassati LR, Piscaglia F, Agnes S, Covino M, et al. Percutaneous ablation procedures in cirrhotic patients with hepatocellular carcinoma submitted to liver transplantation: Assessment of efficacy at explant analysis and of safety for tumor recurrence. Liver Transpl. 2005;11(9):1117-1126.

20. Obara K, Matsumoto N, Okamoto M, Kobayashi M, Ikeda H, Takahashi H, Katakura Y, et al. Insufficient radiofrequency ablation therapy may induce further malignant transformation of hepatocellular carcinoma. Hepatol Int. 2008;2(1):116-123.

21. Yoshida S, Kornek M, Ikenaga N, Schmelzle M, Masuzaki R, Csizmadia E, Wu Y, et al. Sublethal heat treatment promotes epithelial-mesenchymal transition and enhances the malignant potential of hepatocellular carcinoma. Hepatology. 2013;58(5):1667-1680.

22. Dong S, Kong J, Kong F, Gao J, Ke S, Wang S, Ding X, et al. Insufficient radiofrequency ablation promotes epithelial-mesenchymal transition of hepatocellular carcinoma cells through Akt and ERK signaling pathways. J Transl Med. 2013;11:273.

23. Nishihara Y, Aishima S, Kuroda Y, Iguchi T, Taguchi $\mathrm{K}$, Asayama Y, Taketomi A, et al. Biliary phenotype of hepatocellular carcinoma after preoperative transcatheter arterial chemoembolization. J Gastroenterol Hepatol. 2008;23(12):1860-1868. 